\title{
Erratum to: Aflibercept, a New Way to Target Angiogenesis in the Second Line Treatment of Metastatic Colorectal Cancer (mCRC)
}

\author{
Mario Scartozzi $^{1} \cdot$ Loic Vincent $^{2} \cdot$ Marielle Chiron $^{3} \cdot$ Stefano Cascinu $^{4}$
}

Published online: 20 December 2016

(C) Springer International Publishing Switzerland 2016

Erratum to: Targ Oncol (2016) 11:489-500

DOI 10.1007/s11523-016-0447-4

The original version of this article unfortunately contains a mistake.

Page 494, Table 2, 'Characteristic' section, 'Additional eligibility criteria' subsection, ' 1 st line early progressors (1 L PFS $<3$ month)' row: The cell entry in the 'Raise' column, which previously read:

"X"

should read:

" $\checkmark$

The online version of the original article can be found at http://dx.doi. org/10.1007/s11523-016-0447-4.

Mario Scartozzi

marioscartozzi@gmail.com

1 Medical Oncology, University Hospital and University of Cagliari, Pole University Hospital, BDuilio Casula^ - S.S. 554, Km 4,500

Bivio per Sestu, 09042 Monserrato, CA, Italy

2 Sanofi Oncology External Innovation, Vitry-sur-Seine, France

3 Sanofi Oncology Translational Medicine, Vitry-sur-Seine, France

4 Medical Oncology, University Hospital and University of Modena and Reggio Emilia, Modena, Italy 